Bundesgesundheitsbl 2016 · 59:1040-1044

DOI 10.1007/s00103-016-2389-5

(C) Springer-Verlag Berlin Heidelberg 2016
Bekanntmachung des Umweltbundesamtes

\section{Richtwert für Formaldehyd in der Innenraumluft}

\section{Mitteilung des Ausschusses für Innenraumrichtwerte}

\section{Vorbemerkung}

Formaldehyd als gasförmige Substanz führt vorwiegend zu lokalen Reizwirkungen der oberen Atemwege und der Augen. In tierexperimentellen Langzeit-Inhalationsstudien - insbesondere an Ratten traten zytotoxische Wirkungen auf und bei höheren Konzentrationen zeigten sich kanzerogene Effekte mit Tumoren im Nasenbereich. Im Vergleich zu den vorgenannten Gewebsreizungen lassen sich bei deutlich niedrigen Konzentrationen sensorische Reizeffekte an den Schleimhäuten von Nase und Augen beobachten.

Im Juni 2014 wurde Formaldehyd gemäß der 6. Anpassung der CLP-Verordnung in die Gefahrenklassen Karzinogen 1B (wahrscheinlich krebserzeugend beim Menschen; die Einstufung erfolgt überwiegend aufgrund von Nachweisen bei Tieren) und Keimzellmutagen 2 (kann möglicherweise vererbbare Mutationen in Keimzellen beim Menschen auslösen) eingestuft [2]. In den folgenden Abschnitten stellt der Ausschuss für Innenraumrichtwerte die Ergebnisse seiner quantitativen Bewertung der Reizwirkung und des Krebsrisikos durch eingeatmetes Formaldehyd vor.

\section{Stoffidentifizierung [3]}

Systematischer Name: CLP-Index-Nr.: Methanal

EG-Nr.: 605-001-00-5

CAS-Nr.: 200-001-8

Summenformel: 50-00-0 Strukturformel:<smiles>C=O</smiles>

\subsection{Physikalische und chemische Eigenschaften}

Formaldehyd ist eine farblose, hochreaktive, stechend riechende gasförmige Verbindung. In der Umgebungsluft wird es schnell (Halbwertzeit etwa eine Stunde) zu Kohlendioxid oder Methansäure abgebaut [1].

Molekulargewicht: $\quad 30,03 \mathrm{~g} / \mathrm{mol}$

Schmelzpunkt: $\quad-92^{\circ} \mathrm{C}$

Siedepunkt: $\quad-19,1^{\circ} \mathrm{C}$

Wasserlöslichkeit: $\quad 400 \mathrm{~g} / \mathrm{l}\left(\right.$ bei $20^{\circ} \mathrm{C}$ )

Umrechnung $\left(20^{\circ} \mathrm{C}, 1013 \mathrm{hPa}\right)$ :

$1 \mathrm{ml} / \mathrm{m}^{3}=1,249 \mathrm{mg} / \mathrm{m}^{3}$;

$1 \mathrm{mg} / \mathrm{m}^{3}=0,8 \mathrm{ml} / \mathrm{m}^{3}[1]$

\section{Exposition}

Sowohl in der Außenluft als auch in der Innenraumluft wird Formaldehyd bei Verbrennungsvorgängen (z. B. beim Tabakrauchen) freigesetzt. In Innenräumen stellen jedoch Bauprodukte (z. B. Spanplatten, Klebstoffe) sowie Verbraucherprodukte die Hauptquelle für Formaldehyd dar $[1,4]$.

\subsection{Innenraumluft}

Seit mehr als vier Jahrzehnten ist das Vorkommen von Formaldehyd in der Innenraumluft in Deutschland bekannt und untersucht. Neuere Daten sind in $\bullet$ Tab. 1 aufgeführt. Bei einer für Deutschland repräsentativen Untersuchung der Innenraumluft (Kinderumweltsurvey) von Kinderzimmern über 7 Tage fanden sich üblicherweise (95. Perzentil) bis 0,05 $\mathrm{mg}$ Formaldehyd $/ \mathrm{m}^{3}$ und damit etwa halb so hohe Konzentrationen wie 1985/86 [4, 5]. Halbstündige Kurzzeitmessungen in un- gelüfteten Innenräumen ergaben höhere Spitzenkonzentrationen $[6,7]$.

\subsection{Außenluft}

Die Außenluftkonzentrationen in Deutschland liegen meist unter 0,01 mg Formaldehyd $/ \mathrm{m}^{3}[1]$.

\section{Toxikokinetik}

Formaldehyd kommt im Körper als natürliches Stoffwechselprodukt vor und dient als Methyl-Quelle. Die dabei gebildete endogene Formaldehyd-Konzentration im Blut von Säugetieren (Ratte, Primat, Mensch) beträgt 2-3 mg/L Blut [8]. Formaldehyd reagiert im Blut mit basischen Aminogruppen, z. B. der DNA, RNA und Proteinen und bildet u.a. DNA-Protein-Quervernetzungen (DPX), die durch Hydrolyse oder Reparaturvorgänge entfernt werden können. Eine Differenzierung endogener vs. exogener DNA-Formaldehyd-Addukte nach subchronischer Inhalation von ${ }^{13} \mathrm{CD}_{2}$-Formaldehyd in nasalen Epithelzellen von Ratten ergab: im Niedrigdosisbereich fanden sich fast ausschließlich körpereigene Addukte. Der exogene Anteil an DNA-Addukten betrug bei $0,9 \mathrm{mg}$ Formaldehyd $/ \mathrm{m}^{3}$ nur $1 \%$, bei $2,5 \mathrm{mg} / \mathrm{m}^{3} 3 \%$, bei $7,5 \mathrm{mg} / \mathrm{m}^{3} 19 \%$ und bei $12,5 \mathrm{mg} / \mathrm{m}^{3} 40 \%$ [9].

Aus der Luft aufgenommenes Formaldehyd wird aufgrund seiner hohen Wasserlöslichkeit und Reaktivität bei Ratten praktisch vollständig in der Nase resorbiert und verstoffwechselt, bei Primaten und Menschen wird aufgrund ihrer Nasen-Mund-Atmung ein geringer Anteil auch im Rachen, in der Luftröhre und den Bronchien resorbiert [10]. Aus Expositionsmodellen, in denen auch die anatomischen 
Unterschiede des oberen Atemtraktes und die unterschiedlichen Atemraten von Kindern und Erwachsenen berücksichtigt wurden, ergaben sich keine wesentlichen Unterschiede in der inhalativen lokalen Resorptionsrate von Formaldehyd bei Kindern im Vergleich zu Erwachsenen [11, 12].

Ein Weitertransport exogenen Formaldehyds aus dem Nasengewebe bzw. dem oberen Atemtrakt ins Blut findet offenbar nicht in nennenswertem Maße statt: selbst nach 6stündiger Exposition bis zu 12,5 mg ${ }^{13} \mathrm{C}$-Formaldehyd $/ \mathrm{m}^{3}$ zeigt sich keine $\mathrm{Zu}$ nahme der ${ }^{13} \mathrm{C}$-Formaldehyd-Konzentration im Blut von Ratten [13]; die Bestimmungsgrenze für ${ }^{13} \mathrm{C}$-Formaldehyd lag bei $0,03 \mathrm{mg} / \mathrm{l}$ Blut. Ebenso konnten keine exogenen DNA-Formaldehyd-Addukte in entfernteren Geweben wie z. B. dem Knochenmark nachgewiesen werden $[14,15]$.

Formaldehyd reagiert mit Glutathion (GSH) zu GS- $\mathrm{CH}_{2} \mathrm{OH}$. Dieses wird durch das Enzym Formaldehyddehydrogenase (FADH) zu Methansäure und GSH oxidiert. Methansäure wird über den Harn ausgeschieden. Die Methansäureausscheidung weist mit 4-39 mg/d eine hohe Variabilität auf [16] und eignet sich deshalb nicht für ein Biomonitoring einer inhalativen Formaldehyd-Exposition [17].

\section{Gesundheitliche Wirkungen}

Es liegen zahlreiche Übersichtsarbeiten und Bewertungen (z. B. $[1,3,18])$ zu gesundheitlichen Wirkungen eingeatmeten Formaldehyds vor. Die Begründung der Weltgesundheitsorganisation [1] wurde zwischenzeitlich aktualisiert [17]. Im Folgenden werden die für eine regulatorische Bewertung von Formaldehyd relevanten Endpunkte dargestellt. Im Vordergrund stehen hierbei die sensorische Reizwirkung an Auge und Nase sowie die Zytotoxizität und Kanzerogenität.

\subsection{Sensorische Reizung}

Die Reizung der trigeminalen Rezeptoren von Auge und Nasenschleimhaut lässt sich durch Messungen der Blinkfrequenz und Rötung der Konjunktiven sowie des nasalen Flusses und Widerstands objektivieren. In einer Doppelblindstudie an 11 männlichen und 10 weiblichen Probanden mit einer Exposition von jeweils $4 \mathrm{~h} / \mathrm{d}$ an $5 \mathrm{~d} /$ Wo über 2 Wochen gegenüber 0,63 mg Formaldehyd $/ \mathrm{m}^{3}$ (kontinuierlich) oder $0,38 \mathrm{mg} / \mathrm{m}^{3}$ mit Spitzen von $0,75 \mathrm{mg} / \mathrm{m}^{3}$ zeigten sich keine Reizwirkungen (als Blinkfrequenz oder Rötung der Konjunktiven), wohl aber bei 0,63 mg/ $\mathrm{m}^{3}$ mit Spitzen von $1,25 \mathrm{mg} / \mathrm{m}^{3}$ [19]. In einer Folgestudie derselben Arbeitsgruppe an 41 männlichen Probanden mit einer Exposition von jeweils $4 \mathrm{~h} / \mathrm{d}$ an $5 \mathrm{~d}$ gegenüber $0,0,63$ oder $0,88 \mathrm{mg}$ Formaldehyd/ $\mathrm{m}^{3}$ oder gegenüber $0,38 \mathrm{mg} / \mathrm{m}^{3}$ ( $3 \mathrm{~h}, 45^{\prime}$ ) mit Spitzen bei $1,0 \mathrm{mg} / \mathrm{m}^{3}$ (15') wurden keine Reizwirkungen im Sinne einer erhöhten Blinkfrequenz beobachtet [20].

Da Formaldehyd beim Menschen zwar überwiegend im Nasenraum, aber zu einem geringen Anteil auch im oberen Atemtrakt resorbiert wird [10], wurde angesichts der hohen Reaktivität von Formaldehyd in einer Reihe von Studien der Frage nachgegangen, ob es insbesondere bei Kindern Asthma auslösen oder verschlechtern könnte. Insgesamt ergab sich in epidemiologischen Studien an Kindern meist kein hinreichend belastbarer Zusammenhang zwischen einer Formaldehyd-Exposition in der Innenraumluft und der Entwicklung oder Verschlechterung von Asthma [21]. Soweit ein solcher Zusammenhang gefunden wurde, konnte er nicht eindeutig Formaldehyd zugeordnet werden, da die untersuchten Kinder neben Formaldehyd einer Vielzahl weiterer Stoffe bzw. Allergene ausgesetzt waren.

\subsection{Geruchswahrnehmung}

In einer aktuellen Untersuchung an 31 Probanden (15 w, $16 \mathrm{~m}$ ) im Alter von 1835 Jahren ergab sich eine kollektive Geruchswahrnehmungsschwelle $\left(\mathrm{ODT}_{50}\right)$ von $0,14 \mathrm{mg}$ Formaldehyd $/ \mathrm{m}^{3}$ Reinluft [22]. Das 25. Perzentil der kollektiven Geruchswahrnehmungsschwelle lag bei $0,06 \mathrm{mg}$ Formaldehyd $/ \mathrm{m}^{3}$ Reinluft, das 75. und 100. Perzentil bei 0,3 bzw. 0,63 mg Formaldehyd $/ \mathrm{m}^{3}$ Reinluft.

Eine Zusammenstellung früherer Angaben zur Geruchswahrnehmungsschwelle findet sich bei [1].

\subsection{Nasale Zytotoxizität und Zellproliferation}

Bei einer Expositionshöhe um 3 mg Formaldehyd $/ \mathrm{m}^{3}$ ist die nasale Kapazität des Enzyms FADH bei Ratten zur Hälfte gesättigt [23]. Die damit verbundene erhöhte intrazelluläre Formaldehyd-Konzentration wirkt zytotoxisch. Nach chronischer inhalativer Exposition von Ratten gegenüber Formaldehyd traten im vorderen nasalen Bereich (Schnittebene 1 von 5) ab 2,5 mg/m Hyperplasien, Dysplasien, schuppige Metaplasien sowie Entzündungen auf, nicht jedoch bei $1,25 \mathrm{mg} / \mathrm{m}^{3}$ [1]. Ob es sich bei diesen Veränderungen um Tumorvorstufen oder um adaptive Reaktionen handelt, lässt sich nicht sicher beurteilen, da Formaldehyd-bedingte Tumore bei Ratten überwiegend in weiter hinten liegenden Teilen der Nase (Schnittebene 2-3) und erst ab $7 \mathrm{mg} / \mathrm{m}^{3}$ beobachtet wurden [24].

Zur Zellproliferation durch Formaldehyd liegen 8 Studien vor, aus denen sich überwiegend eine niedrigste beobachtete nachteilige Wirkungskonzentration (lowest observed adverse effect concentration - LOAEC) um $7 \mathrm{mg} / \mathrm{m}^{3}$ ergab [24]. In einer dieser Studien stieg nach subchroni-

Tab. 1 Vorkommen von Formaldehyd in der Innenraumluft in Deutschland

\begin{tabular}{|llllllll}
\hline Innenraum & $\mathrm{N}$ & $\mathrm{BG}\left[\mu \mathrm{g} / \mathrm{m}^{3}\right]$ & $\mathrm{N}>\mathrm{BG}(\%)$ & Median $\left[\mu \mathrm{g} / \mathrm{m}^{3}\right]$ & 95. Perz. $\left[\mu \mathrm{g} / \mathrm{m}^{3}\right]$ & Maximum $\left[\mu \mathrm{g} / \mathrm{m}^{3}\right]$ & Zitat \\
\hline $\begin{array}{l}\text { Kinderzimmer, } \\
\begin{array}{l}\text { KUS 2003-2006, } \\
\text { 7d-Passivsammler }\end{array}\end{array}$ & 586 & 1 & $586(100)$ & 24 & 48 & 69 & {$[5]$} \\
\hline $\begin{array}{l}\text { Wohnung etc. 2002-2006, } \\
\text { Kurzzeitmessung, 8 h ungelüftet }\end{array}$ & 446 & $\leq 12$ & $444(99,6)$ & 33 & 105 & 266 & \\
\hline $\begin{array}{l}\text { Wohnung etc. 2006-2012, } \\
\text { Kurzzeitmessung, 8 h ungelüftet }\end{array}$ & 2035 & $\leq 12$ & $1964(96,5)$ & 35 & 113 & 467 & {$[6]$} \\
\hline
\end{tabular}


scher Exposition (6 h/d, 5d/Wo, 13 Wo.) männlicher F344-Ratten gegenüber 0, 0,9, 2,5, 7,5, 12,5 oder $19 \mathrm{mg}$ Formaldehyd $/ \mathrm{m}^{3}$ der Anteil nasaler BrdU-markierten Zellen als Maß der Zellproliferation von $18 \%$ auf $22 \%$, $35 \%$, $38 \%$, $51 \%$ bzw. $64 \%$ [25]. Wegen der niedrigen Anzahl untersuchter Tiere (jeweils $n=5$ ) war der Effekt erst ab 12,5 mg Formaldehyd $/ \mathrm{m}^{3}$ signifikant. Während der Risikobewertungsausschuss der Europäischen Chemikalienagentur [3] die Verdopplung der Proliferationsrate bei $2,5 \mathrm{mg}$ Formaldehyd $/ \mathrm{m}^{3}$ in dieser Studie bereits als LOAEC ansah, wird von anderen Autoren diese als Konzentration ohne beobachtete nachteilige Wirkung (no observed adverse effect concentration - NOAEC) für diesen Endpunkt gewertet [24].

\subsection{Mutagenität und Kanzerogenität}

In verschiedenen in-vitro-Testsystemen erwies sich Formaldehyd als mutagen. Hingegen konnten in-vivo keine eindeutigen gentoxischen Effekte nachgewiesen werden $[1,18]$.

Nach chronischer Inhalation von Formaldehyd (6 h/d, 5 d/Wo, 104 Wochen) zeigten sich in mehreren Studien an Fischer 344-Ratten nasale schuppige Zellkarzinome. Die Expositions-Risiko-Beziehung wies dabei einen nicht-linearen Verlauf auf: die kombinierte Tumorinzidenz betrug bei $2,5 \mathrm{mg}$ Formaldehyd $/ \mathrm{m}^{3}$ 0/364 (0\%), bei 7,5 mg/m 3/325 (1\%), bei $12,5 \mathrm{mg} / \mathrm{m}^{3} 20 / 90$ (22\%) und bei $17,5 \mathrm{mg} /$ $\mathrm{m}^{3} 103 / 232$ (44\%) [17,18]. Mäuse zeigten eine geringere Tumorinzidenz (möglicherweise durch eine verlangsamte Atmung) und werden hier nicht weiter betrachtet.

Ob Formaldehyd auch beim Menschen zu Krebs in den oberen Atemwegen führen kann, lässt sich aus den vorliegenden epidemiologischen Studien an Beschäftigten nicht eindeutig ableiten. In der größten Studie (Kohorte des US-amerikanischen Nationalen Krebsinstituts - NCI) ergab sich eine nicht-signifikant erhöhte Nasen-Rachen-Krebsrate (10 zusätzliche Fälle bei 14.000 Todesfällen von insgesamt 25.600 ehemaligen Beschäftigten) $\mathrm{ab}$ einer durchschnittlichen Konzentration von $1,3 \mathrm{mg} / \mathrm{m}^{3}$ bzw. ab einer Spitzenkonzentration von $5 \mathrm{mg}$ Formaldehyd $/ \mathrm{m}^{3}$

Bundesgesundheitsbl 2016 ·59:1040-1044 DOI 10.1007/s00103-016-2389-5

๑) Springer-Verlag Berlin Heidelberg 2016

Bekanntmachung des Umweltbundesamtes

Richtwert für Formaldehyd in der Innenraumluft. Mitteilung des Ausschusses für Innenraumrichtwerte

\section{Zusammenfassung}

Zum Schutz der Gesundheit der Bevölkerung setzt der Ausschuss für Innenraumrichtwerte Richtwerte für die Innenraumluft fest. Die Reizung der oberen Atemwege beim Menschen sowie eine zytotoxische und tierexperimentell belegte krebserzeugende Wirkung nach langandauernder Einwirkung stellen die kritischen Wirkungsendpunkte eingeatmeten Formaldehyds dar. Für den Endpunkt sensorische Reizwirkung liegen zur Festsetzung eines Richtwertes II (Gefahrenwert) keine belastbaren Angaben für eine LOAEC vor. Ausgehend von einer NOAEC von $0,63 \mathrm{mg} / \mathrm{m}^{3}$ für Reizwirkungen beim Menschen, eines Faktor von 1 für die Zeitextrapolation sowie eines Faktor von 5 für die interindividuelle Variabilität leitet der Ausschuss einen Richtwert I (Vorsorgewert) von $0,1 \mathrm{mg}$ Formaldehyd $/ \mathrm{m}^{3}$ Innenraumluft ab. Nach Auffassung des Ausschusses sollte die Konzentration von 0,1 mg Formaldehyd/ $\mathrm{m}^{3}$ Innenraumluft auch kurzzeitig, bezogen auf einen Messzeitraum von einer halben Stunde, nicht überschritten werden. Zur Abschätzung des Formaldehyd-bedingten Krebsrisikos zieht der Ausschuss angesichts der Ergebnisse der tierexperimentellen Untersuchungen, die auf eine exponentielle Zunahme des Krebsrisiko hinweisen, ein nicht-lineares Modell heran: danach beträgt das zusätzliche theoretische Risiko für einen Nichtraucher, an Krebs der oberen Atemwege zu erkranken, nach kontinuierlicher 80jähriger inhalativer Exposition gegenüber 0,1 mg Formaldehyd $/ \mathrm{m}^{3} 3 \times 10^{-7}$. Damit schützt der Richtwert I auch vor einem Krebsrisiko durch eingeatmetes Formaldehyd.

\section{Schlüsselwörter}

Formaldehyd - Reizwirkung - Kanzerogenität - Risikobewertung - Innenraumluft - Richtwert

\section{Indoor air guide value for formaldehyde. Communication from the Committee on Indoor Guide Values}

\section{Abstract}

The German Committee on Indoor Guide Values issues indoor air guide values to protect public health. Irritation of the human upper airways and cytotoxicity and carcinogenicity in animals studies following chronic exposure are the critical endpoints of inhaled formaldehyde. Regarding sensory irritation no valid LOAEC is available to derive a health hazard guide value (RW II) for formaldehyde in indoor air. Based on a NOAEC of $0.63 \mathrm{mg} /$ $\mathrm{m}^{3}$ for sensory irritation in humans, a factor of 1 for time extrapolation and a factor of 5 for interhuman variability the Committee derives a precautionary indoor air guide value (RW I) of $0.1 \mathrm{mg}$ formaldehyde per cubic meter. The Committee recommends that this guide value should not be exceeded at any interval of half an hour during a day.

For the assessment of the cancer risk of inhaled formaldehyde the Committee uses a non-linear approach due to the results of the animal studies showing an exponential increase of the risk curve: the additional theoretical cancer risk of a non-smoker following a continuous (80 years) inhalative exposure to $0.1 \mathrm{mg}$ formaldehyde per cubic meter is assumed to be $3 \times 10^{-7}$. In conclusion the indoor air guide value for formaldehyde is also protective against cancer risk of inhaled formaldehyde.

\section{Keywords}

Formaldehyde - irritation - carcinogenicity risk evaluation - indoor air - guide value
[26]. Allerdings waren die betroffenen Beschäftigten auch anderen Kanzerogenen ausgesetzt [3].

Leukämie trat in den drei großen epidemiologischen Studien (US-NCI, US-Textilindustrie, UK-Studie) nicht konsistent auf [17]. Im aktuellen Follow-up der zweiten US-Studie hat sich der zunächst beobachtete Zusammenhang zwischen For-
maldehyd-Exposition und Leukämie nicht bestätigt $[27,28]$. Insgesamt wird der $\mathrm{Zu}$ sammenhang als gering erachtet und biologisch als nicht plausibel angesehen [29].

\section{Bewertung}

In Abhängigkeit von der Expositionshöhe lassen sich zwei Wirkungen von Formal- 
dehyd unterscheiden: Im Konzentrationsbereich unter $1 \mathrm{mg} / \mathrm{m}^{3}$ reizt Formaldehyd Auge und Nasenschleimhaut und lässt sich geruchlich wahrnehmen. Die Reizung der trigeminalen Rezeptoren von Auge und Nasenschleimhaut ist in diesem Konzentrationsbereich nicht mit einem histopathologischen Korrelat verknüpft, welches mit zytotoxischen oder kanzerogenen Wirkungen einhergeht.

Bei höheren Konzentrationen nimmt die Kapazität des Enzyms FADH, Formaldehyd zu Methansäure zu oxidieren, ab. Die resultierende erhöhte intrazelluläre Formaldehyd-Konzentration wirkt zytotoxisch. Nach chronischer inhalativer Exposition von Ratten gegenüber Formaldehyd traten in der Nase Hyperplasien, Dysplasien, schuppige Metaplasien sowie Entzündungen auf (LOAEC 2,5 mg/m NOAEC $1,25 \mathrm{mg} / \mathrm{m}^{3}$ ). Etwa ab $7 \mathrm{mg}$ Formaldehyd $/ \mathrm{m}^{3}$ nahmen die Zellproliferation sowie die nasale Krebsinzidenz zu. Die Expositions-Risiko-Beziehung wies dabei einen nicht-linearen Verlauf auf (s. Abschnitt 4.3).

Zur Abschätzung des Formaldehyd-bedingten Krebsrisikos zog die Weltgesundheitsorganisation [1] das nicht-lineare Modell von Conolly et al. [30] heran: danach beträgt das zusätzliche theoretische Risiko für einen Nichtraucher, an Krebs der oberen Atemwege zu erkranken, nach kontinuierlicher 80jähriger inhalativer Exposition gegenüber $0,05 \mathrm{mg}$ Formaldehyd $/ \mathrm{m}^{3} 10^{-7}$, gegenüber $0,1 \mathrm{mg}$ Formaldehyd $/ \mathrm{m}^{3} 3 \times 10^{-7}$ und gegenüber $0,3 \mathrm{mg}$ Formaldehyd $/ \mathrm{m}^{3} 10^{-6}$. Die US-amerikanische Umweltbehörde hat 2010 einen vorläufigen Entwurf zur Abschätzung des Krebsrisikos nach lebenslanger inhalativer Exposition gegenüber Formaldehyd vorgelegt [31]. Der vorläufige Entwurf der US-amerikanischen Umweltbehörde obliegt bislang noch der internen und externen Überprüfung und ist nicht zitierbar.

\subsection{Bestehende Regelungen}

Formaldehyd wurde 2014 gemäß der 6 . Anpassung der CLP-Verordnung (EG) Nr. 1272/2008 auf der Basis von Ergebnissen tierexperimenteller Untersuchungen als wahrscheinlich krebserzeugend beim Menschen (Karzinogen 1 B) und als möglicherweise erbgutverändernd
(Keimzellmutagen 2) eingestuft [2]. Hinsichtlich einer Einstufung von Formaldehyd als krebserzeugend beim Menschen wurden die epidemiologischen Studien an Beschäftigten nicht als hinreichend belastbar angesehen [3]. Die DNA-Addukte und DNA-Protein-Quervernetzungen wurden als indikativ für eine mögliche in-vivo-Mutagenität von Formaldehyd angesehen.

Zum Schutz vor der Reizwirkung sowie vor einem krebserzeugenden Potenzial von Formaldehyd hat der Ausschuss für Gefahrstoffe 2015 einen Arbeitsplatzgrenzwert von $0,37 \mathrm{mg}$ Formaldehyd $/ \mathrm{m}^{3}$ $\left(0,3 \mathrm{ml} / \mathrm{m}^{3}\right)$ festgelegt [18].

Zur Ableitung eines Leitwertes für Formaldehyd in der Innenraumluft ging die Weltgesundheitsorganisation von einer NOAEC von 0,63 mg Formaldehyd $/ \mathrm{m}^{3}$ für den Endpunkt sensorische Reizwirkungen am Auge aus [1]. Für die Zeitextrapolation setzte sie einen Faktor von 1 an, da keine Verstärkung der Effekte mit der Zeit beobachtet worden waren. Ferner sah sie einen Faktor von 5 für die interindividuelle Variabilität als ausreichend an. Daraus ergab sich ein Leitwert von (abgerundet) $0,1 \mathrm{mg}$ Formaldehyd $/ \mathrm{m}^{3}$ Innenraumluft. Nach Ansicht der Weltgesundheitsorganisation sollte diese Konzentration in der Innenraumluft zu keinem Zeitpunkt, bezogen auf einen halbstündigen Messzeitraum, überschritten werden.

\subsection{Richtwert für Formaldehyd in der Innenraumluft}

Der Ausschuss für Innenraumrichtwerte geht gemäß seines Basisschemas zur Ableitung von Richtwerten [32] grundsätzlich vom empfindlichsten Wirkungsendpunkt eines Stoffes aus. Falls sich dabei eine krebserzeugende Wirkung als empfindlichster Endpunkt erweist oder bei einem anderen empfindlichsten Endpunkt eine kanzerogene oder gentoxische Wirkung beteiligt ist, ist die toxikologische Bewertung auf der Grundlage der krebserzeugenden Wirkung vorzunehmen [33]. Als empfindlichste Wirkung des inhalativ aufgenommenen Formaldehyds ist seine Reizwirkung im oberen Atemtrakt anzusehen. Eine zytotoxische und kanzerogene Wirkung wurde erst bei höheren Konzentrationen in tierexperimen- tellen Untersuchungen beobachtet. Vor diesem Hintergrund ist das Krebsrisiko durch eingeatmetes Formaldehyd in dem niedrigen Dosisbereich, in dem Reizwirkungen auftreten, quantitativ zu bewerten.

Zur Bewertung der krebserzeugenden Wirkung eingeatmeten Formaldehyds ging die Weltgesundheitsorganisation von einer nicht-linearen Expositions-Risikobeziehung für die Entstehung von Nasenkrebs nach chronischer Exposition von Ratten gegenüber Formaldehyd aus und zog die Risikoabschätzung von Conolly et al. [30] heran [1]. Danach beträgt das zusätzliche theoretische Krebsrisiko im oberen Atemtrakt für Nichtraucher nach lebenslanger ununterbrochener Exposition (80 Jahre) gegenüber $0,3 \mathrm{mg}$ Formaldehyd $/ \mathrm{m}^{3} 10^{-6}$. Angesichts der Ergebnisse der tierexperimentellen Untersuchungen, die auf eine exponentielle Zunahme des Krebsrisikos hinweisen, schließt sich der Ausschuss für Innenraumrichtwerte der Einschätzung der Weltgesundheitsorganisation an.

Hinsichtlich der nicht-kanzerogenen Wirkung von Formaldehyd ist nach dem Basisschema [32] für die Ableitung eines Richtwertes II (Gefahrenwert) von der niedrigsten beobachteten nachteiligen Wirkungskonzentration auszugehen. Für den Endpunkt sensorische Reizwirkung lässt sich beim derzeitigen Kenntnisstand keine belastbare LOAEC ableiten. Der Ausschuss leitet deshalb nur einen Richtwert I (Vorsorgewert) auf der Basis einer NOAEC ab. In Anlehnung an die Weltgesundheitsorganisation geht der Ausschuss von einer NOAEC von $0,63 \mathrm{mg} / \mathrm{m}^{3}$ für den Endpunkt sensorische Reizwirkungen [19] aus. Angesichts des Fehlens eines histopathologischen Korrelats der trigeminalen Reizwirkung verwendet der Ausschuss einen Faktor von 1 für die Zeitextrapolation sowie nach dem Basisschema [33] einen Faktor von 5 für die interindividuelle Variabilität. Da sich aus anthropometrischen Expositionsmodellen des oberen Atemtraktes von Kinder und Erwachsenen keine wesentlichen Unterschiede in der inhalativen lokalen Resorptionsrate von Formaldehyd bei Kindern im Vergleich zu Erwachsenen [11, 12] ergaben und auch ein Zusammenhang der Entstehung bzw. Verschlimmerung von Asthma bei Kindern bei einer 
Exposition gegenüber Formaldehyd in der Innenraumluft nicht belegt ist [21], verzichtet der Ausschuss auf einen zusätzlichen Faktor im Hinblick auf eine erhöhte Empfindlichkeit von Kindern. Damit ergibt sich ein Richtwert I von 0,63: 1: $5=$ (abgerundet) $0,1 \mathrm{mg}$ Formaldehyd/ $\mathrm{m}^{3}$ Innenraumluft. Angesichts der in Abschnitt 5 genannten Höhe des theoretischen Krebsrisikos schützt dieser Richtwert auch vor einem Krebsrisiko durch eingeatmetes Formaldehyd. Nach Auffassung des Ausschusses sollte eine Konzentration von $0,1 \mathrm{mg}$ Formaldehyd $/ \mathrm{m}^{3}$ Innenraumluft auch kurzzeitig, bezogen auf einen Messzeitraum von einer halben Stunde, nicht überschritten werden, da bei empfindlichen Personen oberhalb dieser Konzentration eine sensorische Reizwirkung auftreten könnte.

\section{Anmerkungen}

Der Entwurf dieser Mitteilung wurde von Dr. Helmut Sagunski und Prof. Dr. Hermann Fromme mit Beiträgen von Dr. Birger Heinzow, Herrn Thomas Lahrz und Dr. Jutta Witten erstellt und im April 2016 vom Ausschuss für Innenraumrichtwerte verabschiedet. Die Literaturrecherche wurde im Dezember 2015 abgeschlossen.

Mit dieser Mitteilung wird die vorangegangene Mitteilung der Ad-hoc-Arbeitsgruppe von 2006 [34] aufgehoben.

\section{Literatur}

1. WHO (2010) Formaldehyde. WHO Guidelines for indoor air quality: selected pollutants. World Health Organization, Genf, S 103-156

2. EU-Komm (2014) Verordnung (EU) Nr. 605/2014 zur Änderung der Verordnung (EG) Nr. 1272/2008 des Europäischen Parlaments und des Rates über die Einstufung, Kennzeichnung und Verpackung von Stoffen und Gemischen zwecks Anpassung an den technischen und wissenschaftlichen Fortschritt. Abl EU L 167/36:1-14 vom 6.6.2014

3. ECHA-RAC (2012) Opinion proposing harmonized classification and labeling at EU level of formaldehyde. European Chemicals Agency. Committee for Risk Assessment. CLH-O-0000003155-80-01/F. Adopted 30 November 2012, Helsinki

4. Salthammer T, Mentese S, Marutzky R (2010) Formaldehyd in the indoor environment. Chem Rev 110:2536-2572

5. UBA (2008) Vergleichswerte für flüchtige organische Verbindungen (VOC und Aldehyde) in der Innenraumluft von Haushalten in Deutschland. Bundesgesundheitsblatt 51:109-112
6. Hofmann $H$, Plieninger $P$ (2008) Bereitstellung einer Datenbank zum Vorkommen von flüchtigen organischen Verbindungen in der Raumluft. Forschungsbericht 20561 234. Umweltbundesamt, Dessau-Roßlau

7. AGÖF (2015) Anhang zum Abschlussbericht des AGÖF-Forschungsprojektes VOC-DB-Datenerhebung. http://www.agoef.de/fileadmin/user upload/dokumente/forschung/AGOEF-Abschlussericht_VOCDB_II_Anhang-nicht-barrierefrei.pdf Letzter Zugriff: 1.12.2015

8. Heck H, Casanova-Schmitz M, Dodd PB et al (1985) Formaldehyde $(\mathrm{CH} 2 \mathrm{O})$ concentrations in the blood of humans and Fischer-344 rats exposed to $\mathrm{CH} 2 \mathrm{O}$ under controlled conditions. Am Ind Hyg Assoc J 46:1-3

9. Lu K, Moeller B, Doyle-Eisele M et al (2011) Molecular dosimetry of N2-hydroxymethyl-dG DNA adducts in rats exposed to formaldehyde. Chem Res Toxicol 24:159-161

10. Schroeter JD, Campbell J, Kimbell JS et al (2014) Effects of endogenous formaldehyde in nasal tissues on inhaled formaldehyde dosimetry predictions in the rat, monkey, and human nasal passages. Toxicol Sci 138:412-424

11. Ginsberg GL, Perkovich Foos B, Firestone MP (2005) Review and analysis of inhalation dosimetry methods for application to children's risk assessment. J Toxicol Environ Health Part A 68:573-615

12. Garcia GJM, Schroeter JD, Segal RA et al (2009) Dosimetry of nasal uptake of water-soluble and reactive gases: a first study of interhuman variability. Inhal Toxicol 21:607-618

13. Kleinnijenhuis AJ, Staal YC, Duistermaat E et al (2013) The determination of exogenous formaldehyde in blood of rats during and after inhalation exposure. Food Chem Toxicol 52:105-112

14. Moeller BC, Lu K, Doyle-Eisele M et al (2011) Determination of N2-hydroxymethyl-dG adducts in the nasal epithelium and bone marrow of nonhuman primates following $13 \mathrm{CD} 2$-formaldehyde inhalation exposure. Chem Res Toxicol 24:162-164

15. Yu R, Lai Y, Hartwell HJ et al (2015) Formation, accumulation, and hydrolysis of endogenous and exogenous formaldehyde-induced DNA damage. Toxicol Sci 146:170-182

16. Berode M, Sethre T, Läubli T, Savolainen H (2000) Urinary methanol and formic acid as indicators of occupational exposure to methyl formiate. Int Arch Occup Environ Health 73:410-414

17. Nielsen, Larsen ST, Wolkoff P (2013) Recent trend in risk assessment of formaldehyde exposures from indoor air. Arch Toxicol 87:73-98

18. AGS (2015) Formaldehyd. Begründung zu Formaldehyd in der TRGS 900. Ausgabe: Februar 2015. Ausschuss für Gefahrstoffe. http://www. baua.de/de/Themen-von-A-Z/Gefahrstoffe/TRGS/ pdf/900/900-formaldehyd.pdf?_blob=publicationFile \&v=2. Letzter Zugriff: 1.12.2015

19. Lang I, Bruckner T, Triebig G (2008) Formaldehyde and chemosensory irritation in humans: a controlled human exposure study. Regul Toxicol Pharmacol 50:23-36

20. Mueller JU, Bruckner T, Triebig G (2013) Exposure study to examine chemosensory effects of formaldehyde on hyposensitive and hypersensitive males. Int Arch Occup Environ Health 86:107-117

21. Fromme H, Sagunski H (2016) Zur Frage eines Asthma auslösenden bzw. verschlechternden Potenzials von Formaldehyd in der Innenraumluft bei Kindern. Bundesgesundheitsblatt : (im Druck)

22. Berglund B, Höglund A, Esfandabad HS (2012) A bisensory method for odour and irritation detec- tion of formaldehyde and pyridine. Chem Percept 5:146-157

23. Andersen ME, Clewell HJ, Bermundez E et al (2010) Formaldehyde: integrating dosimetry, cytotoxicity, and genomics to understand dose-dependent transitions for an endogenous compound. Toxicol Sci 118:716-731

24. Gelbke HP, Gröters S, Morfeld P (2014) Lowest adverse effect concentrations (LOAECs) for formaldehyde exposures. Regul Toxicol Pharmacol 70:340-348

25. Meng F, Bermudez E, McKinzie PB et al (2010) Measurement of tumor-associated mutations in the nasal mucosa of rats exposed to varying doses of formaldehyde. Regul Toxicol Pharmacol 57:274-283

26. Beane Freeman LE, Blair A, Lubin JH et al (2013) Mortality from solid tumors among workers in formaldehyde industries: an update of the $\mathrm{NCl}$ cohort. Am J Ind Med 56:1015-1026

27. Meyers AR, Pinkerton LE, Hein MJ (2013) Cohort mortality study of garment industry workers exposed to formaldehyde: update and internal comparisons. Am J Ind Med 56:1027-1039

28. Checkoway H, Dell LD, Bofetta P et al (2015) Formaldehyde exposure and mortality risks from acute myeloid leukemia and other lymphohematopoietic malignancies in the US National Cancer Institute cohort study of workers in formaldehyde industries. J Occup Environ Med 57:785-794

29. Rhomberg LR, Bailey LA, Goodman JE et al (2011) Is exposure to formaldehyde in air causally associated with leukemia? A hypothesis-based weight-of-evidence analysis. Crit Rev Toxicol 41:555-621

30. Conolly RB, Kimbell JS, Janszen D et al (2004) Human respiratory tract cancer risks of inhaled formaldehyde: dose-response predictions derived from biologically-motivated computational modeling of a combined rodent and human dataset. Toxicol Sci 82:279-296

31. US-EPA (2010) IRIS toxicological review of formaldehyde (inhalation) (external review draft 2010). EPA/635/R-10/002 A. US Environmental Protection Agency, Washington DC

32. Ad-hoc-Arbeitsgruppe Innenraumrichtwerte der IRK / AOLG (2012) Richtwerte für die Innenraumluft: Letzter Zugriff: 1.12.2015

33. AIR (2015) Gesundheitliche Bewertung krebserzeugender Verunreinigungen der Innenraumluft - erste Ergänzung zum Basisschema. Mitteilung des Ausschusses für Innenraumrichtwerte. Bundesgesundheitsblatt 58:769-773

34. Ad-hoc-Arbeitsgruppe der IRK/AOLG (2006) Krebserzeugende Wirkung von Formaldehyd Änderung des Richtwertes für die Innenraumluft von 0,1 ppm nicht erforderlich. Bundesgesundheitsblatt 49:1169 